\title{
Proofs of some partition identities conjectured by Kanade and Russell
}

Hjalmar Rosengren ${ }^{1}$

Dedicated to the memory of Richard Askey

Received: 22 September 2020 / Accepted: 4 January 2021

(c) The Author(s) 2021

\begin{abstract}
Kanade and Russell conjectured several Rogers-Ramanujan-type partition identities, some of which are related to level 2 characters of the affine Lie algebra $A_{9}^{(2)}$. Many of these conjectures have been proved by Bringmann, Jennings-Shaffer and Mahlburg. We give new proofs of five conjectures first proved by those authors, as well as four others that have been open until now. Our proofs for the new cases use quadratic transformations for Askey-Wilson and Rogers polynomials. We also obtain some related results, including a partition identity conjectured by Capparelli and first proved by Andrews.
\end{abstract}

Keywords Kanade-Russell identity · Partition · Rogers polynomial · Askey-Wilson polynomial $\cdot$ Basic hypergeometric series

Mathematics Subject Classification $11 \mathrm{P} 84 \cdot 33 \mathrm{D} 15 \cdot 33 \mathrm{D} 45$

\section{Introduction}

The Rogers-Ramanujan identities are

$$
\sum_{n=0}^{\infty} \frac{q^{n^{2}}}{(q ; q)_{n}}=\frac{1}{\left(q, q^{4} ; q^{5}\right)_{\infty}}, \quad \sum_{n=0}^{\infty} \frac{q^{n(n+1)}}{(q ; q)_{n}}=\frac{1}{\left(q^{2}, q^{3} ; q^{5}\right)_{\infty}}
$$

This work was supported by the Swedish Research Council.

$\bowtie$ Hjalmar Rosengren

hjalmar@chalmers.se

http://www.math.chalmers.se/ hjalmar

1 Department of Mathematical Sciences, Chalmers University of Technology and University of Gothenburg, 41296 Göteborg, Sweden 
Here, we use the standard notation

$$
(a ; q)_{n}=\prod_{j=0}^{n-1}\left(1-a q^{j}\right)
$$

and

$$
\left(a_{1}, \ldots, a_{m} ; q\right)_{n}=\left(a_{1} ; q\right)_{n} \ldots\left(a_{m} ; q\right)_{n},
$$

where $n$ may be infinite. These intriguing identities were first found by Rogers [21] and rediscovered by Ramanujan [20].

The identities (1.1) have a clear combinatorial meaning; for instance, the first identity states that the number of partitions of $n$ without repeated or consecutive parts equals the number of partitions of $n$ into parts congruent to $\pm 1 \bmod 5$. They also have deep connections to affine Lie algebras. Lepowsky and Milne [15] found that the product sides of (1.1) can be interpreted as specialized characters of level 3 modules of the Lie algebra $A_{1}^{(1)}$. Lepowsky and Wilson $[16,17]$ were able to use this fact to give Lie-theoretic proofs of (1.1).

There is now a huge literature on relations between Lie algebras and RogersRamanujan-type identities. Of particular relevance to the present work is Misra's observation [18] that the product sides in (1.1) may alternatively be interpreted as level 2 characters of $A_{7}^{(2)}$. This motivated Kanade and Russell [13] to search for RogersRamanujan-type identities related to level 2 characters of $A_{9}^{(2)}$. They discovered the three conjectured identities (1.3c)-(1.3e) below, which give explicit values of the function

$$
F(u, v, w)=\sum_{i, j, k=0}^{\infty} \frac{(-1)^{k} q^{3 k(k-1)+(i+2 j+3 k)(i+2 j+3 k-1)} u^{i} v^{j} w^{k}}{(q ; q)_{i}\left(q^{4} ; q^{4}\right)_{j}\left(q^{6} ; q^{6}\right)_{k}}
$$

By an extended search, they found six more cases when $F$ appears to have a simple factorization. The resulting nine conjectures are

$$
\begin{aligned}
F\left(q, 1, q^{3}\right) & =\frac{\left(q^{3} ; q^{12}\right)_{\infty}}{\left(q, q^{2} ; q^{4}\right)_{\infty}}, \\
F\left(q^{2}, q^{4}, q^{9}\right) & =\frac{\left(q^{9} ; q^{12}\right)_{\infty}}{\left(q^{2}, q^{3} ; q^{4}\right)_{\infty}}, \\
F\left(q^{4}, q^{6}, q^{15}\right) & =\frac{1}{\left(q^{4}, q^{5}, q^{6}, q^{7}, q^{8} ; q^{12}\right)_{\infty}}, \\
F\left(q, q^{6}, q^{9}\right) & =\frac{1}{\left(q, q^{4}, q^{6}, q^{8}, q^{11} ; q^{12}\right)_{\infty}}, \\
F\left(q^{2}, q^{2}, q^{9}\right) & =\frac{\left(q^{6} ; q^{12}\right)_{\infty}}{\left(q^{2}, q^{3}, q^{4} ; q^{6}\right)_{\infty}},
\end{aligned}
$$




$$
\begin{aligned}
F\left(q^{3}, q^{5}, q^{12}\right) & =\frac{1}{\left(q^{3} ; q^{4}\right)_{\infty}\left(q^{4}, q^{5} ; q^{12}\right)_{\infty}}, \\
F\left(q, q^{3}, q^{6}\right) & =\frac{1}{\left(q ; q^{4}\right)_{\infty}\left(q^{4}, q^{11} ; q^{12}\right)_{\infty}}, \\
F\left(q, q, q^{6}\right) & =\frac{1}{\left(q^{3} ; q^{4}\right)_{\infty}\left(q, q^{8} ; q^{12}\right)_{\infty}}, \\
F\left(q^{2}, q^{-1}, q^{6}\right) & =\frac{1}{\left(q ; q^{4}\right)_{\infty}\left(q^{7}, q^{8} ; q^{12}\right)_{\infty}} ;
\end{aligned}
$$

see Conjecture 5, 5a, 3, 1, 2, 6a, 4, 6 and 4a in [13], respectively. We have stated them more or less in order of increasing difficulty, at least in our approach. For each conjecture, Kanade and Russell gave a combinatorial interpretation in terms of partitions. We stress that [13] contains several other conjectures that will not be discussed here.

The identities (1.3a)-(1.3e) were proved by Bringmann et al. [5]. In the present work, we give a more streamlined proof of those results, and prove the remaining conjectures (1.3f)-(1.3i) for the first time. In each case, we use an integral representation of the triple sum to reduce it to a single sum. The identities (1.3a)-(1.3b) then follow from classical $q$-hypergeometric summation formulas. The identities (1.3c)-(1.3e) are also reduced to classical summations, but we need Watson's quintuple product identity [24] to simplify the result. The identities (1.3f)-(1.3i) are harder since we need one-variable summations that cannot be found in the literature. To prove them we use $q$-orthogonal polynomials. Roughly speaking, we identify the series we wish to sum with generating functions for Askey-Wilson polynomials and then use quadratic transformations, relating Askey-Wilson and Rogers polynomials, to compute them. It is worth mentioning that Rogers polynomials first appeared in Rogers' original proof of (1.1).

The plan of the paper is as follows. After the preliminary Sect. 2, we obtain in Sect. 3 five one-parameter families of reduction formulas, which reduce the triple series $F$ to single series. The left-hand sides of (1.3) all belong to one of these families. In Sect. 4 , we briefly describe how the identities (1.3a)-(1.3e) are obtained in our approach. In Sect. 5, we give some results for other types of multiple series that can be obtained with our methods. In particular, we recover a partition identity related to level 3 characters of $A_{2}^{(2)}$, which was conjectured by Capparelli [6] and first proved by Andrews [1]. The most technical part of the paper is Sect. 6, where we prove (1.3f)-(1.3i), as well as a related identity given in Theorem 5.3.

\section{Preliminaries}

Throughout, $\omega$ is a primitive cubic root of unity and $q$ a number with $0<|q|<1$.

For ease of reference, we recall some classical results for the basic hypergeometric series [8]

$$
{ }_{r} \phi_{s}\left(\begin{array}{l}
a_{1}, \ldots, a_{r} \\
b_{1}, \ldots, b_{s}
\end{array} ;, z\right)=\sum_{n=0}^{\infty} \frac{\left(a_{1}, \ldots, a_{r} ; q\right)_{n}}{\left(b_{1}, \ldots, b_{s} ; q\right)_{n}}\left((-1)^{n} q^{\left(\begin{array}{c}
n \\
2
\end{array}\right)}\right)^{1+s-r} z^{n} .
$$


Namely, we have Euler's $q$-exponential identities

$$
\sum_{n=0}^{\infty} \frac{z^{n}}{(q ; q)_{n}}=\frac{1}{(z ; q)_{\infty}}, \quad \sum_{n=0}^{\infty} \frac{q^{\left(\begin{array}{c}
n \\
2
\end{array}\right) z^{n}}}{(q ; q)_{n}}=(-z ; q)_{\infty}
$$

the $q$-binomial theorem

$$
{ }_{1} \phi_{0}(a ; q, z)=\sum_{n=0}^{\infty} \frac{(a ; q)_{n}}{(q ; q)_{n}} z^{n}=\frac{(a z ; q)_{\infty}}{(z ; q)_{\infty}}
$$

and its disguised version

$$
{ }_{2} \phi_{1}\left(\begin{array}{c}
a,-a \\
-q
\end{array} ; q, z\right)=\sum_{n=0}^{\infty} \frac{\left(a^{2} ; q^{2}\right)_{n}}{\left(q^{2} ; q^{2}\right)_{n}} z^{n}=\frac{\left(a^{2} z ; q^{2}\right)_{\infty}}{\left(z ; q^{2}\right)_{\infty}} .
$$

Applying one of Heine's transformation formulas to (2.3) gives the Bailey-Daum summation

$$
{ }_{2} \phi_{1}\left(\begin{array}{c}
a, b \\
a q / b
\end{array} ; q,-\frac{q}{b}\right)=\frac{(-q ; q)_{\infty}\left(a q, a q^{2} / b^{2} ; q^{2}\right)_{\infty}}{(a q / b,-q / b ; q)_{\infty}} .
$$

The $q$-Gauss summation is

$$
{ }_{2} \phi_{1}\left(\begin{array}{c}
a, b \\
c
\end{array} ; q, \frac{c}{a b}\right)=\frac{(c / a, c / b ; q)_{\infty}}{(c, c / a b ; q)_{\infty}} .
$$

Finally, we mention the transformation

$$
{ }_{2} \phi_{1}\left(\begin{array}{c}
a, b \\
c
\end{array} ; q, z\right)=\frac{(a z, b z ; q)_{\infty}}{(c, z ; q)_{\infty}}{ }_{2} \phi_{2}\left(\begin{array}{c}
z, a b z / c \\
a z, b z
\end{array} ; q, c\right)
$$

which follows by combining [8, (III.2) and (III.4)].

We will need Jacobi's triple product identity

$$
(q, z, q / z ; q)_{\infty}=\sum_{n=-\infty}^{\infty}(-1)^{n} q^{\left(\begin{array}{c}
n \\
2
\end{array}\right)} z^{n}
$$

as well as Watson's quintuple product identity in the form [23, Lemma 6.2]

$\frac{1}{1+z} \frac{\left(z^{2}, z^{-2} ; q^{3}\right)_{\infty}}{\left(z, z^{-1} ; q^{3}\right)_{\infty}}=\frac{1-\omega}{3} \frac{(q ; q)_{\infty}}{\left(q^{3} ; q^{3}\right)_{\infty}}\left(\left(z q \omega, \omega^{2} / z ; q\right)_{\infty}-\omega^{2}\left(z q \omega^{2}, \omega / z ; q\right)_{\infty}\right)$ 
If we replace $q$ by $q^{4}$ and then let $z=q^{-5}, z=q^{-1}$ and $z=q^{-3}$, respectively, we obtain

$$
\begin{aligned}
& \frac{\left(q^{2}, q^{10} ; q^{12}\right)_{\infty}}{\left(q^{4}, q^{5}, q^{7}, q^{8} ; q^{12}\right)_{\infty}}=\frac{\omega-\omega^{2}}{3 q}\left(\left(q \omega, q^{3} \omega^{2} ; q^{4}\right)_{\infty}-\left(q \omega^{2}, q^{3} \omega ; q^{4}\right)_{\infty}\right), \\
& \frac{\left(q^{2}, q^{10} ; q^{12}\right)_{\infty}}{\left(q, q^{4}, q^{8}, q^{11} ; q^{12}\right)_{\infty}}=\frac{1-\omega^{2}}{3}\left(\left(q \omega, q^{3} \omega^{2} ; q^{4}\right)_{\infty}-\omega\left(q \omega^{2}, q^{3} \omega ; q^{4}\right)_{\infty}\right), \\
& \frac{\left(q^{6} ; q^{12}\right)_{\infty}^{2}}{\left(q^{3}, q^{4}, q^{8}, q^{9} ; q^{12}\right)_{\infty}}=\frac{1-\omega}{3}\left(\left(q \omega, q^{3} \omega^{2} ; q^{4}\right)_{\infty}-\omega^{2}\left(q \omega^{2}, q^{3} \omega ; q^{4}\right)_{\infty}\right) .
\end{aligned}
$$

\section{Reduction from triple to single series}

In [5], Bringmann et al. showed that the nine conjectures (1.3) can be reduced to sums of single series, which they could evaluate in the first five cases. In this section, we will obtain more general reductions using the following integral representation of the function (1.2).

Proposition 3.1 We have

$$
F(u, v, w)=\left(q^{2} ; q^{2}\right)_{\infty} \oint \frac{\left(1 / z, q^{2} z ; q^{2}\right)_{\infty}\left(-w z^{3} ; q^{6}\right)_{\infty}}{(-u z ; q)_{\infty}\left(v z^{2} ; q^{4}\right)_{\infty}} \frac{\mathrm{d} z}{2 \pi \mathrm{i} z}
$$

where the integration is over a positively oriented contour separating 0 from all poles of the integrand.

Proof We may take the contour close enough to zero so that $|u z|<1$ and $\left|v z^{2}\right|<1$. By (2.1) and (2.7), the right-hand side of (3.1) equals

$$
\oint \sum_{i=0}^{\infty} \frac{(-u z)^{i}}{(q ; q)_{i}} \sum_{j=0}^{\infty} \frac{\left(v z^{2}\right)^{j}}{\left(q^{4} ; q^{4}\right)_{j}} \sum_{k=0}^{\infty} \frac{q^{3 k(k-1)}\left(w z^{3}\right)^{k}}{\left(q^{6} ; q^{6}\right)_{k}} \sum_{l=-\infty}^{\infty} q^{l(l-1)}\left(-z^{-1}\right)^{l} \frac{\mathrm{d} z}{2 \pi \mathrm{i} z} .
$$

The integration picks out the constant term in this Laurent series, that is, the term with $l=i+2 j+3 k$.

We will also need the following integral representation of the function ${ }_{2} \phi_{1}$. Although it is an easy consequence of known results, we have not found it in the literature.

Proposition 3.2 For $|t|<1$, we have the contour integral representation

$$
{ }_{2} \phi_{1}\left(\begin{array}{c}
a, b \\
c
\end{array} ; q, t\right)=\frac{(q ; q)_{\infty}}{(c, t ; q)_{\infty}} \oint \frac{(a b z, c z, q z / t, t / z ; q)_{\infty}}{(a z, b z, c z / t ; q)_{\infty}} \frac{\mathrm{d} z}{2 \pi \mathrm{i} z}
$$


where the integral is over a positively oriented contour separating 0 from all poles of the integrand.

Note that the right-hand side displays the $S_{3} \times S_{2}$-symmetry of the function ${ }_{2} \phi_{1}$ implied by Heine's transformations [8, (III.1)-(III.3)]. Moreover, it gives the analytic continuation of the left-hand side to $t \in \mathbb{C} \backslash q^{\mathbb{Z}_{\leq 0}}$.

Proof We start from the more general integral

$$
I=\oint \frac{(a b z, c z, q z / t, t / z ; q)_{\infty}}{(a z, b z, c z / t, d / z ; q)_{\infty}} \frac{\mathrm{d} z}{2 \pi \mathrm{i} z}
$$

which has an additional sequence of poles at $\left(d q^{k}\right)_{k=0}^{\infty}$. We take $d$ close to zero so that all those poles are inside the contour. If $|t|<|d|$, then by [8, Eq. (4.10.8)] the integral equals the sum of the residues inside the contour, which can be written

$$
\frac{(a b d, c d, d q / t, t / d ; q)_{\infty}}{(q, a d, b d, c d / t ; q)_{\infty}}{ }_{3} \phi_{2}\left(\begin{array}{c}
a d, b d, c d / t \\
a b d, c d
\end{array} ; q, \frac{t}{d}\right)
$$

Applying the transformation formula [8, Eq. (III.9)] gives

$$
I=\frac{(a b d, d q / t, t, c ; q)_{\infty}}{(q, a d, b d, c d / t ; q)_{\infty}} 3 \phi_{2}\left(\begin{array}{c}
a, b, c d / t \\
c, a b d
\end{array} ; q, t\right) .
$$

By analytic continuation, this holds for $|t|<1$. We now obtain the desired result by letting $d \rightarrow 0$.

Replacing $z$ by $t z$ and relabelling the parameters gives the equivalent identity

$$
\begin{aligned}
\oint & \frac{\left(\alpha_{1} z, \alpha_{2} z, q z, 1 / z ; q\right)_{\infty}}{\left(\beta_{1} z, \beta_{2} z, \beta_{3} z ; q\right)_{\infty}} \frac{\mathrm{d} z}{2 \pi \mathrm{i} z} \\
= & \frac{\left(\beta_{1}, \alpha_{1} / \beta_{1} ; q\right)_{\infty}}{(q ; q)_{\infty}}{ }_{2} \phi_{1}\left(\begin{array}{c}
\alpha_{2} / \beta_{2}, \alpha_{2} / \beta_{3} \\
\beta_{1}
\end{array} q, \frac{\alpha_{1}}{\beta_{1}}\right), \quad \alpha_{1} \alpha_{2}=\beta_{1} \beta_{2} \beta_{3} .
\end{aligned}
$$

This can be compared with (3.1), where the integrand can be written

$$
\frac{\left(-w^{1 / 3} z,-w^{1 / 3} \omega z,-w^{1 / 3} \omega^{2} z, q^{2} z, 1 / z ; q^{2}\right)_{\infty}}{\left(-u z,-u q z, v^{1 / 2} z,-v^{1 / 2} z ; q^{2}\right)_{\infty}} .
$$

We obtain an integral of the form (3.2) (with $q$ replaced by $q^{2}$ ) if $w^{1 / 3} \in\left\{u, u q, v^{1 / 2}\right\}$ and in addition $w=u^{2} v q$. That is, there are three one-parameter cases where $F$ can be reduced to the function ${ }_{2} \phi_{1}$. 
Proposition 3.3 We have the reduction identities

$$
\begin{aligned}
F\left(u, u q^{-1}, u^{3}\right)= & \left(-u^{1 / 2} q^{-1 / 2}, u^{1 / 2} q^{1 / 2} \omega^{2} ; q^{2}\right)_{\infty} \\
& \times{ }_{2} \phi_{1}\left(\begin{array}{c}
q^{-1} \omega,-u^{1 / 2} q^{1 / 2} \omega \\
-u^{1 / 2} q^{-1 / 2} ; q^{2}, u^{1 / 2} q^{1 / 2} \omega^{2}
\end{array}\right), \\
F\left(u, u q^{2}, u^{3} q^{3}\right)= & \left(-u, q \omega^{2} ; q^{2}\right)_{\infty} 2 \phi_{1}\left(\begin{array}{c}
u^{1 / 2} \omega,-u^{1 / 2} \omega \\
-u
\end{array} q^{2}, q \omega^{2}\right), \\
F\left(u, u^{4} q^{2}, u^{6} q^{3}\right) & =\left(-u q, u \omega^{2} ; q^{2}\right)_{\infty} \phi_{1}\left(\begin{array}{c}
-\omega, u q \omega \\
-u q
\end{array} q^{2}, u \omega^{2}\right),
\end{aligned}
$$

assuming that the ${ }_{2} \phi_{1}$-series converge.

Applying (2.6) to (3.2) gives the equivalent identity

$$
\begin{aligned}
\oint & \frac{\left(\alpha_{1} z, \alpha_{2} z, q z, 1 / z ; q\right)_{\infty}}{\left(\beta_{1} z, \beta_{2} z, \beta_{3} z ; q\right)_{\infty}} \frac{\mathrm{d} z}{2 \pi \mathrm{i} z} \\
= & \frac{\left(\beta_{2}, \beta_{3} ; q\right)_{\infty}}{(q ; q)_{\infty}} 2 \phi_{2}\left(\begin{array}{c}
\alpha_{1} / \beta_{1}, \alpha_{2} / \beta_{1} \\
\beta_{2}, \beta_{3}
\end{array} ;, \beta_{1}\right), \quad \alpha_{1} \alpha_{2}=\beta_{1} \beta_{2} \beta_{3} .
\end{aligned}
$$

This leads to alternative representations for (3.3) as ${ }_{2} \phi_{2}$-series, which have infinite radius of convergence and hence hold for all $u$.

The Kanade-Russell conjectures (1.3a)-(1.3d) and (1.3f)-(1.3g) are all reduced to ${ }_{2} \phi_{1}$-evaluations by Proposition 3.3. In the remaining three conjectures, $(1.3 \mathrm{e})$ and (1.3h)-(1.3i), the variables in $F(u, v, w)$ satisfy $w=u^{2} v q^{3}$ rather than $w=u^{2} v q$. They can be expressed as integrals of the form (3.2) with $\alpha_{1} \alpha_{2}=\beta_{1} \beta_{2} \beta_{3} q$. It is easy to see that any such integral can be expressed (in several different ways) as the sum of two integrals with $\alpha_{1} \alpha_{2}=\beta_{1} \beta_{2} \beta_{3}$, and hence as a sum of two $2 \phi_{1}$-series. If $\beta_{3}=-\beta_{2}$, there is a more compact expression as $\mathrm{a}_{2} \phi_{2}$-series.

Lemma 3.4 When $\alpha_{1} \alpha_{2}=-\beta_{1} \beta_{2}^{2} q$, then

$$
\oint \frac{\left(\alpha_{1} z, \alpha_{2} z, q z, 1 / z ; q\right)_{\infty}}{\left(\beta_{1} z, \beta_{2} z,-\beta_{2} z ; q\right)_{\infty}} \frac{\mathrm{d} z}{2 \pi \mathrm{i} z}=\frac{\left(\beta_{2}^{2} q^{2} ; q^{2}\right)_{\infty}}{(q ; q)_{\infty}}{ }_{2} \phi_{2}\left(\begin{array}{c}
\alpha_{1} / \beta_{1}, \alpha_{2} / \beta_{1} \\
\beta_{2} q,-\beta_{2} q
\end{array} ; q, \beta_{1}\right) .
$$

Proof Let $I$ denote the given integral. Inserting the factor

$$
1=\frac{\left(1+\beta_{2} z\right)+\left(1-\beta_{2} z\right)}{2}
$$

it splits as a sum, where we may evaluate each term using (3.4). This gives

$$
\begin{aligned}
I & =\frac{1}{2} \oint \frac{\left(\alpha_{1} z, \alpha_{2} z, q z, 1 / z ; q\right)_{\infty}}{\left(\beta_{1} z, \beta_{2} z,-\beta_{2} q z ; q\right)_{\infty}} \frac{\mathrm{d} z}{2 \pi \mathrm{i} z}+\frac{1}{2} \oint \frac{\left(\alpha_{1} z, \alpha_{2} z, q z, 1 / z ; q\right)_{\infty}}{\left(\beta_{1} z, \beta_{2} q z,-\beta_{2} z ; q\right)_{\infty}} \frac{\mathrm{d} z}{2 \pi \mathrm{i} z} \\
& =\frac{1}{2(q ; q)_{\infty}}\left(\left(\beta_{2},-\beta_{2} q ; q\right)_{\infty} 2 \phi_{2}\left(\begin{array}{c}
\alpha_{1} / \beta_{1}, \alpha_{2} / \beta_{1} \\
\beta_{2},-\beta_{2} q
\end{array} ;, \beta_{1}\right)\right.
\end{aligned}
$$




$$
\left.+\left(\beta_{2} q,-\beta_{2} ; q\right)_{\infty} 2 \phi_{2}\left(\begin{array}{c}
\alpha_{1} / \beta_{1}, \alpha_{2} / \beta_{1} \\
\beta_{2} q,-\beta_{2}
\end{array} ; q, \beta_{1}\right) \cdot\right)
$$

Adding the series termwise gives

$I=\frac{\left(\beta_{2}^{2} q^{2} ; q^{2}\right)_{\infty}}{(q ; q)_{\infty}} \sum_{k=0}^{\infty} \frac{\left(\alpha_{1} / \beta_{1}, \alpha_{2} / \beta_{1} ; q\right)_{k}}{\left(\beta_{2} q,-\beta_{2} q ; q\right)_{k}}(-1)^{k} q^{\left(\begin{array}{c}k \\ 2\end{array}\right)} \beta_{1}^{k} \frac{\left(1-\beta_{2} q^{k}\right)+\left(1+\beta_{2} q^{k}\right)}{2}$

which simplifies to the desired expression.

Combining Proposition 3.1 and Lemma 3.4 gives the following reduction identities. Note that (1.3e) and (1.3h) are series of the form (3.5a) whereas (1.3i) is of the form (3.5b).

Proposition 3.5 We have

$$
\begin{aligned}
& F\left(u, u, u^{3} q^{3}\right)=\left(u q^{4} ; q^{4}\right)_{\infty}{ }^{2} \phi_{2}\left(\begin{array}{c}
q \omega, q \omega^{2} \\
u^{1 / 2} q^{2},-u^{1 / 2} q^{2}
\end{array} q^{2},-u\right), \\
& F\left(u, u q^{-3}, u^{3}\right)=\left(u q ; q^{4}\right)_{\infty} 2 \phi_{2}\left(\begin{array}{c}
q^{-1} \omega, q^{-1} \omega^{2} \\
u^{1 / 2} q^{1 / 2},-u^{1 / 2} q^{1 / 2} ; q^{2},-u q
\end{array}\right) .
\end{aligned}
$$

\section{Results of Bringmann, Jennings-Shaffer and Mahlburg}

For completeness, we show how to prove the conjectures (1.3a)-(1.3e) in our approach. Bringmann et al. [5] also obtain these results by reducing triple summations to single summations, which are either the same as ours or easily seen to be equivalent. The main virtue of our approach is the more streamlined way to obtain such reductions as explained in Sect. 3.

Proof of (1.3a). If we let $u=q$ in (3.3a) we get

$$
\begin{aligned}
F\left(q, 1, q^{3}\right) & =\left(-1, q \omega^{2} ; q^{2}\right)_{\infty}{ }_{2} \phi_{1}\left(\begin{array}{c}
q^{-1} \omega,-q \omega \\
-1
\end{array} ; q^{2}, q \omega^{2}\right) \\
& =\left(-q^{2} ; q^{2}\right)_{\infty}\left(q \omega, q \omega^{2} ; q^{4}\right)_{\infty},
\end{aligned}
$$

where we used the Bailey-Daum summation (2.4). This can be rewritten as (1.3a). Proof of (1.3b). If we let $u=q^{2}$ in (3.3b), we get

$$
\begin{aligned}
F\left(q^{2}, q^{4}, q^{9}\right) & =\left(-q^{2}, q \omega^{2} ; q^{2}\right)_{\infty}{ }_{2} \phi_{1}\left(\begin{array}{c}
q \omega,-q \omega \\
-q^{2}
\end{array} ; q^{2}, q \omega^{2}\right) \\
& =\left(-q^{2} ; q^{2}\right)_{\infty}\left(q^{3} \omega, q^{3} \omega^{2} ; q^{4}\right)_{\infty},
\end{aligned}
$$

by either (2.3) or (2.4). This can be written as (1.3b).

Proof of (1.3c). If we let $u=q^{4}$ in (3.3b), we get

$$
F\left(q^{4}, q^{6}, q^{15}\right)=\left(-q^{4}, q \omega^{2} ; q^{2}\right)_{\infty} 2 \phi_{1}\left(\begin{array}{c}
q^{2} \omega,-q^{2} \omega \\
-q^{4}
\end{array} q^{2}, q \omega^{2}\right) .
$$


This ${ }_{2} \phi_{1}$ is contiguous to a ${ }_{1} \phi_{0}$ in base $q^{4}$. More precisely, the terms can be expressed as

$$
\frac{\left(q^{2} \omega,-q^{2} \omega ; q^{2}\right)_{k}}{\left(q^{2},-q^{4} ; q^{2}\right)_{k}}\left(q \omega^{2}\right)^{k}=\frac{\omega\left(1+q^{2}\right)}{\left(1-\omega^{2}\right) q} \frac{\left(\omega^{2} ; q^{4}\right)_{k+1}}{\left(q^{4} ; q^{4}\right)_{k+1}}\left(q \omega^{2}\right)^{k}\left(1-q^{2 k}\right) .
$$

Replacing $k$ by $k-1$ we find that

$$
\begin{aligned}
F\left(q^{4}, q^{6}, q^{15}\right) & =\frac{\omega\left(-q^{2}, q \omega^{2} ; q^{2}\right)_{\infty}}{\left(1-\omega^{2}\right) q}\left({ }_{1} \phi_{0}\left(\omega^{2} ; q^{4}, q \omega^{2}\right)-{ }_{1} \phi_{0}\left(\omega^{2} ; q^{4}, q^{3} \omega^{2}\right)\right) \\
& =\frac{\omega\left(-q^{2} ; q^{2}\right)_{\infty}}{\left(1-\omega^{2}\right) q}\left(\left(q \omega, q^{3} \omega^{2} ; q^{4}\right)_{\infty}-\left(q \omega^{2}, q^{3} \omega ; q^{4}\right)_{\infty}\right)
\end{aligned}
$$

where we used the $q$-binomial theorem (2.2). Applying (2.8a) and simplifying we get (1.3c).

Proof of (1.3d). If we let $u=q$ in (3.3c) we get

$$
F\left(q, q^{6}, q^{9}\right)=\left(-q^{2}, q \omega^{2} ; q^{2}\right)_{\infty}{ }^{2} \phi_{1}\left(\begin{array}{l}
q^{2} \omega,-\omega \\
-q^{2}
\end{array} q^{2}, q \omega^{2}\right) .
$$

This time we write the terms as

$$
\frac{\left(q^{2} \omega,-\omega ; q^{2}\right)_{k}}{\left(q^{2},-q^{2} ; q^{2}\right)_{k}}\left(q \omega^{2}\right)^{k}=\frac{1}{1-\omega} \frac{\left(\omega^{2} ; q^{4}\right)_{k}}{\left(q^{4} ; q^{4}\right)_{k}}\left(q \omega^{2}\right)^{k}\left(1-\omega q^{2 k}\right),
$$

which gives

$$
\begin{aligned}
F\left(q, q^{6}, q^{9}\right) & =\frac{\left(-q^{2}, q \omega^{2} ; q^{2}\right)_{\infty}}{1-\omega}\left({ }_{1} \phi_{0}\left(\omega^{2} ; q^{4}, q \omega^{2}\right)-\omega_{1} \phi_{0}\left(\omega^{2} ; q^{4}, q^{3} \omega^{2}\right)\right) \\
& =\frac{\left(-q^{2} ; q^{2}\right)_{\infty}}{(1-\omega)}\left(\left(q \omega, q^{3} \omega^{2} ; q^{4}\right)_{\infty}-\omega\left(q \omega^{2}, q^{3} \omega ; q^{4}\right)_{\infty}\right) .
\end{aligned}
$$

Applying (2.8b) we arrive at (1.3d).

Proof of (1.3e). Although $F\left(q^{2}, q^{2}, q^{9}\right)$ is a special case of (3.5a), we do not use that identity. It is easier to start from (3.1), which gives

$$
F\left(q^{2}, q^{2}, q^{9}\right)=\left(q^{2} ; q^{2}\right)_{\infty} \oint \frac{\left(-q^{3} \omega z,-q^{3} \omega^{2} z, 1 / z, q^{2} z ; q^{2}\right)_{\infty}}{\left(q z,-q z,-q^{2} z ; q^{2}\right)_{\infty}} \frac{\mathrm{d} z}{2 \pi \mathrm{i} z}
$$

Inserting the factor

$$
1=\frac{(1+q \omega z)-\omega^{2}\left(1+q \omega^{2} z\right)}{1-\omega^{2}}
$$


gives

$$
\begin{aligned}
& F\left(q^{2}, q^{2}, q^{9}\right)=\frac{\left(q^{2} ; q^{2}\right)_{\infty}}{1-\omega^{2}}\left(\oint \frac{\left(-q \omega z,-q^{3} \omega^{2} z, 1 / z, q^{2} z ; q^{2}\right)_{\infty}}{\left(q z,-q z,-q^{2} z ; q^{2}\right)_{\infty}} \frac{\mathrm{d} z}{2 \pi \mathrm{i} z}\right. \\
& \left.-\omega^{2} \oint \frac{\left(-q^{3} \omega z,-q \omega^{2} z, 1 / z, q^{2} z ; q^{2}\right)_{\infty}}{\left(q z,-q z,-q^{2} z ; q^{2}\right)_{\infty}} \frac{\mathrm{d} z}{2 \pi \mathrm{i} z}\right) \\
& =\frac{\left(-q^{2} ; q^{2}\right)_{\infty}}{1-\omega^{2}}\left(\left(q \omega^{2} ; q^{2}\right)_{\infty} 2 \phi_{1}\left(\begin{array}{c}
\omega,-\omega \\
-q^{2}
\end{array} ; q^{2}, q \omega^{2}\right)\right. \\
& -\omega^{2}\left(q \omega ; q^{2}\right)_{\infty 2} \phi_{1}\left(\begin{array}{c}
\omega^{2},-\omega^{2} \\
\left.\left.-q^{2} ; q^{2}, q \omega\right)\right)
\end{array}\right. \\
& =\frac{\left(-q^{2} ; q^{2}\right)_{\infty}}{1-\omega^{2}}\left(\left(q \omega, q^{3} \omega^{2} ; q^{4}\right)_{\infty}-\omega^{2}\left(q \omega^{2}, q^{3} \omega ; q^{4}\right)_{\infty}\right) \text {, }
\end{aligned}
$$

where we used first (3.2) and then (2.3). Applying (2.8c) we arrive at (1.3e).

\section{Other types of multiple series}

The integral (3.2) can be used to study other types of multiple series apart from $F$. We will give some examples that seem very close to the identities (1.3). We first observe that (3.2) can be evaluated by (2.3) (or, alternatively, (2.4)) whenever $\beta_{1}=-q$ and $\beta_{2}=-\beta_{3}$. Indeed, assuming also $\left|\alpha_{1}\right|<q$ we have

$$
\begin{aligned}
& \oint \frac{\left(\alpha_{1} z, \alpha_{2} z, q z, 1 / z ; q\right)_{\infty}}{\left(-q z, \beta_{2} z,-\beta_{2} z ; q\right)_{\infty}} \frac{\mathrm{d} z}{2 \pi \mathrm{i} z} \\
& \quad=\frac{\left(-q,-\alpha_{1} q^{-1} ; q\right)_{\infty}}{(q ; q)_{\infty}}{ }_{2} \phi_{1}\left(\begin{array}{c}
\alpha_{2} / \beta_{2},-\alpha_{2} / \beta_{2} \\
-q
\end{array} ;,-\alpha_{1} q^{-1}\right) \\
& \quad=\frac{(-q ; q)_{\infty}\left(-\alpha_{1},-\alpha_{2} ; q^{2}\right)_{\infty}}{(q ; q)_{\infty}}, \quad \alpha_{1} \alpha_{2}=\beta_{2}^{2} q .
\end{aligned}
$$

By analytic continuation, the final expression is valid for general values of $\alpha_{1}$. This particular case of Proposition 3.2 is equivalent to [7, Eq. (2.1)].

We will only consider (5.1) in the special case $\alpha_{2}=\omega \alpha_{1}$. Replacing $q$ by $q^{2}$, it can be written

$$
\oint \frac{\left(-u^{3} q^{3} z^{3} ; q^{6}\right)_{\infty}\left(q^{2} z, 1 / z ; q^{2}\right)_{\infty}}{\left(-q^{2} z, u z,-u z,-u q z ; q^{2}\right)_{\infty}} \frac{\mathrm{d} z}{2 \pi \mathrm{i} z}=\frac{\left(-q^{2} ; q^{2}\right)_{\infty}\left(u^{3} q^{3} ; q^{12}\right)_{\infty}}{\left(q^{2} ; q^{2}\right)_{\infty}\left(u q ; q^{4}\right)_{\infty}}
$$

Let us write

$$
f(u)=\left(-q^{2} z, u z,-u z,-u q z ; q^{2}\right)_{\infty}
$$


for the denominator on the left-hand side. We may obtain double or triple summations by combining factors from $f$ before expanding the integrand as a Laurent series. The most compact expression is obtained for $u=-q$, since

$$
f(-q)=\left(q z,-q z, q^{2} z,-q^{2} z ; q^{2}\right)_{\infty}=\left(q^{2} z^{2} ; q^{2}\right)_{\infty}
$$

By (2.1) and (2.7), the left-hand side of (5.2) then takes the form

$$
\begin{aligned}
\oint & \frac{\left(q^{6} z^{3} ; q^{6}\right)_{\infty}\left(q^{2} z, 1 / z ; q^{2}\right)_{\infty}}{\left(q^{2} z^{2} ; q^{2}\right)_{\infty}} \frac{\mathrm{d} z}{2 \pi \mathrm{i} z} \\
= & \frac{1}{\left(q^{2} ; q^{2}\right)_{\infty}} \sum_{j, k=0}^{\infty} \sum_{l=-\infty}^{\infty} \frac{(-1)^{k+l} q^{2 j+6\left(\begin{array}{c}
k \\
2
\end{array}\right)+6 k+2\left(\begin{array}{c}
l \\
2
\end{array}\right)}}{\left(q^{2} ; q^{2}\right)_{j}\left(q^{6} ; q^{6}\right)_{k}} \oint z^{2 j+3 k-l} \frac{\mathrm{d} z}{2 \pi \mathrm{i} z} \\
= & \frac{1}{\left(q^{2} ; q^{2}\right)_{\infty}} \sum_{j, k=0}^{\infty} \frac{q^{4 j^{2}+12 j k+12 k^{2}}}{\left(q^{2} ; q^{2}\right)_{j}\left(q^{6} ; q^{6}\right)_{k}} .
\end{aligned}
$$

After replacing $q^{2}$ by $q$ we obtain the following double summation. It was shown in $[13,14]$ that it is equivalent to a partition identity conjectured by Capparelli [6] and first proved by Andrews [1]. As the resulting proof of Caparelli's identity is based on (5.1), it is very close to the proof of a more general result given in [7].

Proposition 5.1 (Andrews, Kanade-Russell, Kurşungöz) We have

$$
\sum_{j, k=0}^{\infty} \frac{q^{2 j^{2}+6 j k+6 k^{2}}}{(q ; q)_{j}\left(q^{3} ; q^{3}\right)_{k}}=\frac{1}{\left(q^{3} ; q^{6}\right)_{\infty}\left(q^{2}, q^{10} ; q^{12}\right)_{\infty}}
$$

If we can write $f(u)$ as the product of two factors of the form $\left(a z^{k} ; \pm q^{l}\right)_{\infty}$, we obtain in the same way a triple summation. In Sect. 4 , we obtained (1.3a) from the factorization

$$
f(1)=\left(z,-z,-q z,-q^{2} z ; q^{2}\right)_{\infty}=(-q z ; q)_{\infty}\left(z^{2} ; q^{4}\right)_{\infty}
$$

and (1.3b) from

$$
f\left(q^{2}\right)=\left(q^{2} z,-q^{2} z,-q^{2} z,-q^{3} z ; q^{2}\right)_{\infty}=\left(-q^{2} z ; q\right)_{\infty}\left(q^{4} z^{2} ; q^{4}\right)_{\infty} .
$$

There are essentially three other cases when this can be done, namely,

$$
\begin{aligned}
f\left(-q^{3}\right) & =\left(-q^{2} z, q^{3} z,-q^{3} z, q^{4} z\right)=\left(-q^{2} z ; q\right)_{\infty}\left(q^{3} z ; q\right)_{\infty}, \\
f(q) & =\left(q z,-q z,-q^{2} z,-q^{2} z ; q^{2}\right)_{\infty}=(-q z ; q)_{\infty}(q z ;-q)_{\infty}, \\
f\left(q^{3}\right) & =\left(-q^{2} z, q^{3} z,-q^{3} z,-q^{4} z ; q^{2}\right)_{\infty}=\left(-q^{2} z ; q\right)_{\infty}\left(q^{3} z ;-q\right)_{\infty} .
\end{aligned}
$$


The corresponding three summations are as follows. To our knowledge, they are new. We do not know whether they have natural interpretations as partition identities, nor whether they are related to Lie algebras.

Theorem 5.2 We have

$$
\begin{aligned}
\sum_{i, j, k=0}^{\infty} \frac{(-1)^{j} q^{2\left(\begin{array}{c}
i+j+3 k \\
2
\end{array}\right)+6\left(\begin{array}{l}
k \\
2
\end{array}\right)+2 i+3 j+12 k}}{(q ; q)_{i}(q ; q)_{j}\left(q^{6} ; q^{6}\right)_{k}} & =\frac{\left(q^{4}, q^{20} ; q^{24}\right)_{\infty}}{\left(q^{2} ; q^{4}\right)_{\infty}} \\
\sum_{i, j, k=0}^{\infty} \frac{(-1)^{j+k} q^{2\left({ }^{i+j+3 k}\right)+6\left(\begin{array}{l}
k \\
2
\end{array}\right)+i+j+6 k}}{(q ; q)_{i}(-q ;-q)_{j}\left(q^{6} ; q^{6}\right)_{k}} & =\frac{1}{\left(q^{2} ; q^{4}\right)_{\infty}\left(q^{2}, q^{10} ; q^{12}\right)_{\infty}} \\
\sum_{i, j, k=0}^{\infty} \frac{(-1)^{j+k} q^{2\left({ }^{i+j+3 k}\right)+6\left(\begin{array}{l}
k \\
2
\end{array}\right)+2 i+3 j+12 k}}{(q ; q)_{i}(-q ;-q)_{j}\left(q^{6} ; q^{6}\right)_{k}} & =\frac{\left(q^{12} ; q^{12}\right)_{\infty}}{\left(q^{2} ; q^{2}\right)_{\infty}}
\end{aligned}
$$

We have found one more result for the type of triple series appearing in (5.3b)(5.3c). The proof, which is parallel to that of (1.3f), is given in Sect. 6 .

Theorem 5.3 We have

$$
\begin{aligned}
& \sum_{i, j, k=0}^{\infty} \frac{(-1)^{j+k} q^{2\left(\begin{array}{c}
i+j+3 k \\
2
\end{array}\right)+6\left(\begin{array}{l}
k \\
2
\end{array}\right)+2 i+\frac{3}{2} j+9 k}}{(q ; q)_{i}(-q ;-q)_{j}\left(q^{6} ; q^{6}\right)_{k}} \\
& =\frac{1}{\left(-q^{3 / 2}, q^{5 / 2} ;-q^{3}\right)_{\infty}\left(q^{4} ; q^{6}\right)_{\infty}\left(q^{2} ; q^{12}\right)_{\infty}}
\end{aligned}
$$

\section{New cases of the Kanade-Russell conjectures}

In this section, we prove the conjectures (1.3f)-(1.3i) as well as (5.4).

\subsection{Reduction to one-variable series}

We first reduce the results to one-variable summations. By (3.3b) and (3.5) we have the reduction formulas

$$
\begin{gathered}
F\left(q^{3}, q^{5}, q^{12}\right)=\left(-q^{3}, q \omega^{2} ; q^{2}\right)_{\infty 2} \phi_{1}\left(\begin{array}{c}
q^{3 / 2} \omega,-q^{3 / 2} \omega \\
-q^{3}
\end{array} q^{2}, q \omega^{2}\right) \\
F\left(q, q^{3}, q^{6}\right)=\left(-q, q \omega^{2} ; q^{2}\right)_{\infty 2} \phi_{1}\left(\begin{array}{c}
q^{1 / 2} \omega,-q^{1 / 2} \omega \\
-q
\end{array} q^{2}, q \omega^{2}\right) \\
F\left(q, q, q^{6}\right)=\left(q^{5} ; q^{4}\right)_{\infty 2} \phi_{2}\left(\begin{array}{c}
q \omega, q \omega^{2} \\
q^{5 / 2},-q^{5 / 2} ; q^{2},-q
\end{array}\right), \\
F\left(q^{2}, q^{-1}, q^{6}\right)=\left(q^{3} ; q^{4}\right)_{\infty 2} \phi_{2}\left(\begin{array}{c}
q^{-1} \omega, q^{-1} \omega^{2} \\
q^{3 / 2},-q^{3 / 2} ; q^{2},-q^{3}
\end{array}\right) .
\end{gathered}
$$


For the first two cases, Bringmann et al. [5] found equivalent reductions but for the last two cases, their reductions are more complicated. Replacing $q$ by $q^{1 / 2}$, it follows that the conjectures (1.3f)-(1.3i) are equivalent to the following summations.

Theorem 6.1 The following summation formulas hold:

$$
\begin{aligned}
& { }_{2} \phi_{1}\left(\begin{array}{c}
q^{3 / 4} \omega,-q^{3 / 4} \omega \\
-q^{3 / 2}
\end{array} ; q, q^{1 / 2} \omega^{2}\right)=\frac{\left(1+q^{1 / 2}\right)\left(q^{1 / 2}, q^{9 / 2} ; q^{6}\right)_{\infty}}{\left(q^{1 / 2} \omega^{2} ; q\right)_{\infty}\left(q ; q^{2}\right)_{\infty}\left(q^{2} ; q^{6}\right)_{\infty}}, \\
& { }_{2} \phi_{1}\left(\begin{array}{c}
q^{1 / 4} \omega,-q^{1 / 4} \omega \\
-q^{1 / 2}
\end{array} ; q, q^{1 / 2} \omega^{2}\right)=\frac{\left(q^{3 / 2}, q^{7 / 2} ; q^{6}\right)_{\infty}}{\left(q^{1 / 2} \omega^{2} ; q\right)_{\infty}\left(q ; q^{2}\right)_{\infty}\left(q^{2} ; q^{6}\right)_{\infty}}, \\
& { }_{2} \phi_{2}\left(\begin{array}{c}
q^{1 / 2} \omega, q^{1 / 2} \omega^{2} \\
q^{5 / 4},-q^{5 / 4}
\end{array} ; q,-q^{1 / 2}\right)=\frac{1-q^{1 / 2}}{\left(q^{1 / 2} ; q\right)_{\infty}\left(q^{1 / 2}, q^{4} ; q^{6}\right)_{\infty}}, \\
& { }_{2} \phi_{2}\left(\begin{array}{c}
q^{-1 / 2} \omega, q^{-1 / 2} \omega^{2} \\
q^{3 / 4},-q^{3 / 4}
\end{array} q,-q^{3 / 2}\right)=\frac{1}{\left(q^{1 / 2} ; q\right)_{\infty}\left(q^{7 / 2}, q^{4} ; q^{6}\right)_{\infty}} .
\end{aligned}
$$

The sum in (5.4) can be represented as

$$
\begin{gathered}
\left(q^{2} ; q^{2}\right)_{\infty} \oint \frac{\left(-q^{9} z^{3} ; q^{6}\right)_{\infty}\left(1 / z, q^{2} z ; q^{2}\right)_{\infty}}{\left(-q^{2} z ; q\right)_{\infty}\left(q^{3 / 2} z ;-q\right)_{\infty}} \frac{\mathrm{d} z}{2 \pi \mathrm{i} z} \\
=\left(q^{2} ; q^{2}\right)_{\infty} \oint \frac{\left(-q^{3} \omega z,-q^{3} \omega^{2} z, 1 / z, q^{2} z ; q^{2}\right)_{\infty}}{\left(-q^{2} z, q^{3 / 2} z,-q^{5 / 2} z ; q^{2}\right)_{\infty}} \frac{\mathrm{d} z}{2 \pi \mathrm{i} z} \\
=\left(-q^{2}, q \omega^{2} ; q^{2}\right)_{\infty 2} \phi_{1}\left(\begin{array}{c}
q^{1 / 2} \omega,-q^{3 / 2} \omega \\
-q^{2}
\end{array} q^{2}, q \omega^{2}\right)
\end{gathered}
$$

where we used (3.2). It follows that Theorem 5.3 is equivalent to the following result.

Theorem 6.2 We have the summation formula

$$
{ }_{2} \phi_{1}\left(\begin{array}{c}
q^{1 / 4} \omega,-q^{3 / 4} \omega \\
-q
\end{array} ; q, q^{1 / 2} \omega^{2}\right)=\frac{\left(q^{3} ; q^{6}\right)_{\infty}}{\left(q^{1 / 2} \omega^{2} ; q\right)_{\infty}\left(-q^{3 / 4}, q^{5 / 4} ;-q^{3 / 2}\right)_{\infty}\left(q^{2} ; q^{6}\right)_{\infty}} .
$$

\subsection{Orthogonal polynomials}

Our main tool to prove Theorems 6.1 and 6.2 is quadratic transformations relating Askey-Wilson and Rogers polynomials. From now onwards, the variables $x$ and $z$ are related by

$$
x=\frac{z+z^{-1}}{2} .
$$

The Rogers (or continuous $q$-ultraspherical) polynomials [2,22] are given by the generating function

$$
\frac{(a t z, a t / z ; q)_{\infty}}{(t z, t / z ; q)_{\infty}}=\sum_{n=0}^{\infty} C_{n}(x ; a \mid q) t^{n}
$$


The more general Askey-Wilson polynomials [3] have the generating function [11]

$$
{ }_{2} \phi_{1}\left(\begin{array}{c}
a z, b z \\
a b
\end{array} ; q, \frac{t}{z}\right){ }_{2} \phi_{1}\left(\begin{array}{c}
c / z, d / z \\
c d
\end{array} ; q, t z\right)=\sum_{n=0}^{\infty} \frac{p_{n}(x ; a, b, c, d \mid q)}{(q, a b, c d ; q)_{n}} t^{n}
$$

These polynomials are symmetric in the parameters $(a, b, c, d)$. The series in (6.3) and (6.4) converge for $|t|<\min \left(|z|,|z|^{-1}\right)$.

The Rogers polynomials appear as a special case of the Askey-Wilson polynomials in several different ways [8, Sect. 7.5]. For instance,

$$
\begin{aligned}
C_{n}\left(x ; a^{2} \mid q\right) & =\frac{\left(a^{4} ; q\right)_{n}}{\left(q,-a^{2}, a^{2} q^{1 / 2},-a^{2} q^{1 / 2} ; q\right)_{n}} p_{n}\left(x ; a,-a, a q^{1 / 2},-a q^{1 / 2} \mid q\right) \\
C_{n}\left(x ; a^{2} \mid q^{2}\right) & =\frac{\left(a^{2} ; q\right)_{n}}{\left(q^{2}, a^{2} q ; q^{2}\right)_{n}} p_{n}\left(x ; a,-a, q^{1 / 2},-q^{1 / 2} \mid q\right) \\
C_{2 n}(x ; a \mid q) & =\frac{\left(a^{2} ; q^{2}\right)_{n}}{(q,-a ; q)_{2 n}} p_{n}\left(2 x^{2}-1 ; a, a q,-1,-q \mid q^{2}\right) \\
C_{2 n+1}(x ; a \mid q) & =\frac{2\left(a^{2} ; q^{2}\right)_{n+1}}{(q,-a ; q)_{2 n+1}} x p_{n}\left(2 x^{2}-1 ; a, a q,-q,-q^{2} \mid q^{2}\right)
\end{aligned}
$$

These relations are easy to understand by comparing the orthogonality measures for the polynomials involved.

Using (6.5) in (6.4) leads to alternative generating functions for Rogers polynomials. In particular, (6.5a) gives

$$
\begin{gathered}
{ }_{2} \phi_{1}\left(\begin{array}{c}
a z,-a z \\
-a^{2} ; q, \frac{t}{z}
\end{array}\right){ }_{2} \phi_{1}\left(\begin{array}{c}
a q^{1 / 2} / z,-a q^{1 / 2} / z \\
-a^{2} q
\end{array} c^{\infty}, t z\right) \\
\quad=\sum_{n=0}^{\infty} \frac{\left(a^{2} q^{1 / 2},-a^{2} q^{1 / 2} ; q\right)_{n}}{\left(-a^{2} q, a^{4} ; q\right)_{n}} C_{n}\left(x ; a^{2} \mid q\right) t^{n}
\end{gathered}
$$

The generating functions arising from $(6.5 \mathrm{~b})-(6.5 \mathrm{~d})$ are as follows.

Lemma 6.3 The Rogers polynomials have the generating functions

$$
\begin{aligned}
\frac{\left(t q / z ; q^{2}\right)_{\infty}}{\left(t z ; q^{2}\right)_{\infty}}{ }_{2} \phi_{1}\left(\begin{array}{c}
a z,-a z \\
-a^{2}
\end{array} ; q, \frac{t}{z}\right) & =\sum_{n=0}^{\infty} \frac{\left(a^{2} q ; q^{2}\right)_{n}}{\left(a^{4} ; q^{2}\right)_{n}} C_{n}\left(x ; a^{2} \mid q^{2}\right) t^{n}, \\
\frac{(-t / z ; q)_{\infty}}{(t z ; q)_{\infty}}{ }_{2} \phi_{1}\left(\begin{array}{c}
a z^{2}, a z^{2} q \\
a^{2} q
\end{array} ; q^{2}, \frac{t^{2}}{z^{2}}\right) & =\sum_{n=0}^{\infty} \frac{(-a ; q)_{n}}{\left(a^{2} ; q\right)_{n}} C_{n}(x ; a \mid q) t^{n},
\end{aligned}
$$

where the right-hand sides converge for $|t|<\min \left(|z|,|z|^{-1}\right)$. 
Proof Choose $(a, b, c, d)=\left(a,-a, q^{1 / 2},-q^{1 / 2}\right)$ in (6.4). By (6.5b), the right-hand side can be identified with the right-hand side of (6.7a). On the left-hand side, we apply (2.3) in the form

$$
{ }_{2} \phi_{1}\left(\begin{array}{c}
q^{1 / 2} / z,-q^{1 / 2} / z \\
-q
\end{array} ;, t z\right)=\frac{\left(t q / z ; q^{2}\right)_{\infty}}{\left(t z ; q^{2}\right)_{\infty}}
$$

This proves (6.7a).

Using (6.5c)-(6.5d) and (6.4), the right-hand side of (6.7b) can be written

$$
\begin{aligned}
\sum_{n=0}^{\infty} & \frac{t^{2 n}}{\left(q, q^{2}, a^{2} q ; q^{2}\right)_{n}} p_{n}\left(2 x^{2}-1 ; a, a q,-1,-q \mid q^{2}\right) \\
& +\frac{2 t x}{1-q} \sum_{n=0}^{\infty} \frac{t^{2 n}}{\left(q^{2}, q^{3}, a^{2} q ; q^{2}\right)_{n}} p_{n}\left(2 x^{2}-1 ; a, a q,-q,-q^{2} \mid q^{2}\right) \\
= & { }_{2} \phi_{1}\left(\begin{array}{c}
a z^{2}, a z^{2} q \\
a^{2} q
\end{array} q^{2}, \frac{t^{2}}{z^{2}}\right)\left(2 \phi_{1}\left(\begin{array}{c}
\left.-1 / z^{2},-q / z^{2} ; q^{2}, t^{2} z^{2}\right) \\
q
\end{array}\right)\right. \\
& \left.+\frac{2 t x}{1-q} 2 \phi_{1}\left(\begin{array}{c}
-q / z^{2},-q^{2} / z^{2} \\
q^{3}
\end{array} q^{2}, t^{2} z^{2}\right)\right) .
\end{aligned}
$$

The final factor is evaluated by the $q$-binomial theorem in the form

$$
\begin{aligned}
& \sum_{n=0}^{\infty} \frac{\left(-1 / z^{2} ; q\right)_{2 n}}{(q ; q)_{2 n}}(t z)^{2 n}+\sum_{n=0}^{\infty} \frac{\left(-1 / z^{2} ; q\right)_{2 n+1}}{(q ; q)_{2 n+1}}(t z)^{2 n+1} \\
& \quad=\sum_{n=0}^{\infty} \frac{\left(-1 / z^{2} ; q\right)_{n}}{(q ; q)_{n}}(t z)^{n}=\frac{(-t / z ; q)_{\infty}}{(t z ; q)_{\infty}}
\end{aligned}
$$

This proves (6.7b).

Applying (2.6) to the left-hand side of (6.7a) gives the equivalent identity

$$
\begin{gathered}
\frac{\left(a^{2} t^{2} ; q^{2}\right)_{\infty}}{\left(-a^{2} ; q\right)_{\infty}\left(t z, t / z ; q^{2}\right)_{\infty}} 2 \phi_{2}\left(\begin{array}{c}
t z, t / z \\
a t,-a t
\end{array} ; q,-a^{2}\right) \\
=\sum_{n=0}^{\infty} \frac{\left(a^{2} q ; q^{2}\right)_{n}}{\left(a^{4} ; q^{2}\right)_{n}} C_{n}\left(x ; a^{2} \mid q^{2}\right) t^{n} .
\end{gathered}
$$

To prove (1.3i) we will need the following variation of (6.9).

Lemma 6.4 The Rogers polynomials have the generating function

$$
\frac{\left(a^{2} t^{2} ; q^{2}\right)_{\infty}}{\left(-a^{2} q^{-1} ; q\right)_{\infty}\left(t z, t / z ; q^{2}\right)_{\infty}} 2 \phi_{2}\left(\begin{array}{c}
t z, t / z \\
a t,-a t
\end{array} ; q,-a^{2} q^{-1}\right)
$$




$$
=\sum_{n=0}^{\infty} \frac{\left(a^{2} q^{-1} ; q^{2}\right)_{n}}{\left(a^{4} q^{-2} ; q^{2}\right)_{n}} C_{n}\left(x ; a^{2} \mid q^{2}\right) t^{n},
$$

where the right-hand side converges for $|t|<\min \left(|z|,|z|^{-1}\right)$.

Proof We start from the identity

$$
\begin{aligned}
p_{n}\left(x ; a q^{-1},-a, q^{1 / 2},-q^{1 / 2} \mid q\right)= & \frac{1-a^{2} q^{n-1}}{1-a^{2} q^{2 n-1}} p_{n}\left(x ; a,-a, q^{1 / 2},-q^{1 / 2} \mid q\right) \\
& -a q^{-1}\left(1-q^{2 n}\right) p_{n-1}\left(x ; a,-a, q^{1 / 2},-q^{1 / 2} \mid q\right),
\end{aligned}
$$

which is a special case of [8, Eq. (7.6.8)] (in the first edition, a factor $(q ; q)_{n}$ is missing on the right-hand side of (7.6.9)). Taking the even part in $a$ gives

$$
\begin{aligned}
& p_{n}\left(x ; a q^{-1},-a, q^{1 / 2},-q^{1 / 2} \mid q\right)+p_{n}\left(x ; a,-a q^{-1}, q^{1 / 2},-q^{1 / 2} \mid q\right) \\
& \quad=2 \frac{1-a^{2} q^{n-1}}{1-a^{2} q^{2 n-1}} p_{n}\left(x ; a,-a, q^{1 / 2},-q^{1 / 2} \mid q\right)=2 \frac{\left(q^{2}, a^{2} q^{-1} ; q^{2}\right)_{n}}{\left(a^{2} q^{-1} ; q\right)_{n}} C_{n}\left(x ; a^{2} \mid q^{2}\right),
\end{aligned}
$$

where we used (6.5b). It follows that the right-hand side of (6.10) equals

$$
\frac{1}{2} \sum_{n=0}^{\infty} \frac{p_{n}\left(x ; a q^{-1},-a, q^{1 / 2},-q^{1 / 2} \mid q\right)+p_{n}\left(x ; a,-a q^{-1}, q^{1 / 2},-q^{1 / 2} \mid q\right)}{\left(q,-a^{2} q^{-1},-q ; q\right)_{n}} t^{n} .
$$

Summing the series using (6.4) and simplifying using (6.8) gives

$$
\frac{1}{2} \frac{\left(t q / z ; q^{2}\right)_{\infty}}{\left(t z ; q^{2}\right)_{\infty}}\left({ }_{2} \phi_{1}\left(\begin{array}{c}
a q^{-1} z,-a z \\
-a^{2} q^{-1} ; q, \frac{t}{z}
\end{array}\right)+{ }_{2} \phi_{1}\left(\begin{array}{c}
a z,-a q^{-1} z \\
-a^{2} q^{-1} ; q, \frac{t}{z}
\end{array}\right)\right) .
$$

By (2.6), this is equal to

$$
\begin{aligned}
& \frac{\left(a^{2} t^{2} ; q^{2}\right)_{\infty}}{2\left(t z, t / z ; q^{2}\right)_{\infty}\left(-a^{2} q^{-1} ; q\right)_{\infty}}\left(\left(1-a t q^{-1}\right)_{2} \phi_{2}\left(\begin{array}{c}
t z, t / z \\
a t q^{-1},-a t
\end{array} ; q,-a^{2} q^{-1}\right)\right. \\
& \left.+\left(1+a t q^{-1}\right)_{2} \phi_{2}\left(\begin{array}{c}
t z, t / z \\
a t,-a t q^{-1}
\end{array} ; q,-a^{2} q^{-1}\right)\right) .
\end{aligned}
$$

Adding the series termwise as in the proof of Lemma 3.4 gives the desired expression.

Another key tool is the following identity for Rogers polynomials at $x=-1 / 2$ or, equivalently, $z=\omega$.

Lemma 6.5 One has

$$
C_{n}\left(-\frac{1}{2} ; a \mid q\right)=\sum_{l=0}^{\lfloor n / 3\rfloor} \frac{\left(a^{3} ; q^{3}\right)_{l}\left(a^{-1} ; q\right)_{n-3 l}}{\left(q^{3} ; q^{3}\right)_{l}(q ; q)_{n-3 l}} a^{n-3 l} .
$$


To prove this, we simply use the $q$-binomial theorem to write the generating function (6.3) as

$$
\begin{aligned}
\frac{\left(a t \omega, a t \omega^{-1} ; q\right)_{\infty}}{\left(t \omega, t \omega^{-1} ; q\right)_{\infty}} & =\frac{(t ; q)_{\infty}}{(a t ; q)_{\infty}} \cdot \frac{\left(a^{3} t^{3} ; q^{3}\right)_{\infty}}{\left(t^{3} ; q^{3}\right)_{\infty}} \\
& =\sum_{k=0}^{\infty} \frac{\left(a^{-1} ; q\right)_{k}}{(q ; q)_{k}}(a t)^{k} \sum_{l=0}^{\infty} \frac{\left(a^{3} ; q^{3}\right)_{l}}{\left(q^{3} ; q^{3}\right)_{l}} t^{3 l}
\end{aligned}
$$

In hypergeometric notation, (6.11) is

$$
C_{n}\left(-\frac{1}{2} ; a \mid q\right)=\frac{a^{n}\left(a^{-1} ; q\right)_{n}}{(q ; q)_{n}}{ }_{4} \phi_{3}\left(\begin{array}{c}
q^{-n}, q^{1-n}, q^{2-n}, a^{3} \\
\left.a q^{1-n}, a q^{2-n}, a q^{3-n} ; q^{3}, q^{3}\right)
\end{array}\right.
$$

This is a balanced series, so one can obtain alternative expressions by applying Sears' and Watson's transformation formulas to the right-hand side.

Combining the results stated above, we obtain the following sextic transformations, which seem to be new.

Proposition 6.6 The transformation formulas

$$
\begin{aligned}
& { }_{2} \phi_{1}\left(\begin{array}{c}
a \omega,-a \omega \\
-a^{2} ; q, a^{2} q^{-1} \omega^{2}
\end{array}\right)=\frac{\left(a^{6} ; q^{2}\right)_{\infty}\left(a^{6} q^{-3} ; q^{6}\right)_{\infty}}{\left(a^{2} q^{-1} \omega^{2}, a^{4} q^{-1} ; q\right)_{\infty}} \\
& { }_{3} \phi_{2}\left(\begin{array}{l}
a^{2} q, a^{2} q^{3}, a^{2} q^{5} ; q^{6}, a^{6} q^{-3} \\
a^{6} q^{2}, a^{6} q^{4}
\end{array}\right), \\
& { }_{2} \phi_{1}\left(\begin{array}{c}
a \omega, a q \omega \\
a^{2} q
\end{array} ; q^{2}, a^{2} \omega^{2}\right)=\frac{\left(a^{3} ; q\right)_{\infty}\left(-a^{3} ; q^{3}\right)_{\infty}}{\left(a^{2} \omega^{2}, a^{4} ; q^{2}\right)_{\infty}} \\
& { }_{3} \phi_{2}\left(\begin{array}{l}
-a,-a q,-a q^{2} \\
a^{3} q, a^{3} q^{2}
\end{array} ;-a^{3}\right),
\end{aligned}
$$

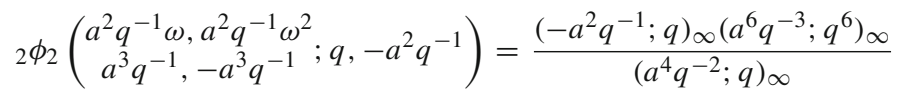

$$
\begin{aligned}
& { }_{3} \phi_{2}\left(\begin{array}{c}
a^{2} q^{-1}, a^{2} q, a^{2} q^{3} \\
a^{6} q^{-2}, a^{6} q^{2}
\end{array} q^{6}, a^{6} q^{-3}\right),
\end{aligned}
$$

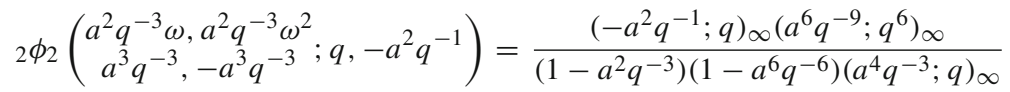

$$
\begin{aligned}
& \times\left({ }_{3} \phi_{2}\left(\begin{array}{c}
a^{2} q^{-1}, a^{2} q, a^{2} q^{3} \\
a^{6} q^{-4}, a^{6} q^{-2} ; q^{6}, a^{6} q^{-9}
\end{array}\right)\right. \\
& -a^{2} q^{-3} \frac{1-a^{2} q^{-1}}{1-a^{6} q^{-4}} \\
& \left.{ }_{3} \phi_{2}\left(\begin{array}{c}
a^{2} q, a^{2} q^{3}, a^{2} q^{5} \\
a^{6} q^{-2}, a^{6} q^{2}
\end{array} ; q^{6}, a^{6} q^{-9}\right)\right),
\end{aligned}
$$

hold when the series on both sides converge. 
Proof We first consider (6.12c). We let $z=\omega$ in (6.10), insert the first expression in Lemma 6.5 , replace $n$ by $n+3 l$ and change the order of summation. This gives

$$
\begin{aligned}
& \frac{\left(a^{2} t^{2} ; q^{2}\right)_{\infty}}{\left(-a^{2} q^{-1} ; q\right)_{\infty}\left(t \omega, t \omega^{2} ; q^{2}\right)_{\infty}}{ }_{2} \phi_{2}\left(\begin{array}{c}
t \omega, t \omega^{2} \\
a t,-a t
\end{array} ;,-a^{2} q^{-1}\right) \\
& =\sum_{l=0}^{\infty} \sum_{n=0}^{\infty} \frac{\left(a^{2} q^{-1} ; q^{2}\right)_{n+3 l}\left(a^{6} ; q^{6}\right)_{l}\left(a^{-2} ; q^{2}\right)_{n}}{\left(a^{4} q^{-2} ; q^{2}\right)_{n+3 l}\left(q^{6} ; q^{6}\right)_{l}\left(q^{2} ; q^{2}\right)_{n}} a^{2 n} t^{n+3 l} \\
& =\sum_{l=0}^{\infty} \frac{\left(a^{2} q^{-1} ; q^{2}\right)_{3 l}\left(a^{6} ; q^{6}\right)_{l}}{\left(a^{4} q^{-2} ; q^{2}\right)_{3 l}\left(q^{6} ; q^{6}\right)_{l}} t_{2 \phi_{1}}^{3 l}\left(\begin{array}{c}
a^{-2}, a^{2} q^{6 l-1} \\
\left.a^{4} q^{6 l-2} ; q^{2}, a^{2} t\right) .
\end{array}\right.
\end{aligned}
$$

We now specialize $t=a^{2} q^{-1}$ and apply the $q$-Gauss summation (2.5) in the form

$$
{ }_{2} \phi_{1}\left(\begin{array}{c}
a^{-2}, a^{2} q^{6 l-1} \\
a^{4} q^{6 l-2}
\end{array} ; q^{2}, a^{4} q^{-1}\right)=\frac{\left(a^{6} q^{-2}, a^{2} q^{-1} ; q^{2}\right)_{\infty}}{\left(a^{4} q^{-2} ; q\right)_{\infty}} \frac{\left(a^{4} q^{-2} ; q^{2}\right)_{3 l}}{\left(a^{6} q^{-2} ; q^{2}\right)_{3 l}}
$$

It is straight-forward to write the result as in $(6.12 \mathrm{c})$.

The identities (6.12a) and (6.12b) follow in exactly the same way from (6.7a) and (6.7b), respectively. We do not give the details.

Finally, we let $t=a^{2} q^{-3}$ in (6.13) and use [8, (III.2)] to write

$$
\begin{aligned}
& { }_{2} \phi_{1}\left(\begin{array}{c}
a^{-2}, a^{2} q^{6 l-1} \\
a^{4} q^{6 l-2} ; q^{2}, a^{4} q^{-3}
\end{array}\right)=\frac{\left(a^{2} q^{-1}, a^{6} q^{6 l-4} ; q^{2}\right)_{\infty}}{\left(a^{4} q^{-3}, a^{4} q^{6 l-2} ; q^{2}\right)_{\infty}} \\
& { }_{2} \phi_{1}\left(\begin{array}{c}
q^{-2}, a^{2} q^{6 l-1} \\
a^{6} q^{6 l-4} ; q^{2}, a^{2} q^{-1}
\end{array}\right) \\
& =\frac{\left(a^{2} q^{-1}, a^{6} q^{6 l-4} ; q^{2}\right)_{\infty}}{\left(a^{4} q^{-3}, a^{4} q^{6 l-2} ; q^{2}\right)_{\infty}} \\
& \left(1-a^{2} q^{-3} \frac{1-a^{2} q^{6 l-1}}{1-a^{6} q^{6 l-4}}\right) \text {. }
\end{aligned}
$$

Inserting this into (6.13) and simplifying gives (6.12d).

\subsection{Proof of the remaining triple summations}

We are now ready to prove Theorems 6.1 and 6.2, which imply the four KanadeRussell conjectures (1.3f)-(1.3i) and the new summation (5.4).

Proof of (1.3f). Let $a=q^{3 / 4}$ in (6.12a). Then, the right-hand side reduces to

$$
\frac{\left(q^{9 / 2} ; q^{2}\right)_{\infty}\left(q^{3 / 2} ; q^{6}\right)_{\infty}}{\left(q^{1 / 2} \omega^{2} ; q\right)_{\infty}\left(q^{2} ; q\right)_{\infty}}{ }_{2} \phi_{1}\left(\begin{array}{c}
q^{5 / 2}, q^{9 / 2} \\
q^{17 / 2} ; q^{6}, q^{3 / 2}
\end{array}\right)
$$

Applying the $q$-Gauss summation (2.5) and simplifying gives (6.1a). 
Proof of (1.3g). We need the generating function (6.6). Like in the proof of Proposition 6.6, we let $z=\omega$, insert the first expression in Lemma 6.5 and change the order of summation. This gives

$$
\begin{aligned}
{ }_{2} \phi_{1} & \left(\begin{array}{c}
a \omega,-a \omega \\
-a^{2} ; q, \frac{t}{\omega}
\end{array}\right){ }_{2} \phi_{1}\left(\begin{array}{c}
a q^{1 / 2} \omega^{2},-a q^{1 / 2} \omega^{2} \\
-a^{2} q
\end{array} ; q, t \omega\right) \\
= & \sum_{l=0}^{\infty} \frac{\left(a^{2} q^{1 / 2},-a^{2} q^{1 / 2} ; q\right)_{3 l}\left(a^{6} ; q^{3}\right)_{l}}{\left(-a^{2} q, a^{4} ; q\right)_{3 l}\left(q^{3} ; q^{3}\right)_{l}} t^{3 l} \\
& \times{ }_{3} \phi_{2}\left(\begin{array}{c}
a^{-2}, a^{2} q^{3 l+1 / 2},-a^{2} q^{3 l+1 / 2} \\
-a^{2} q^{3 l+1}, a^{4} q^{3 l} ; q, a^{2} t
\end{array}\right) .
\end{aligned}
$$

In the special case $a=q^{1 / 4}$ and $t=q^{1 / 2}$, the inner series reduces to

$$
2 \phi_{1}\left(\begin{array}{c}
q^{-1 / 2},-q^{3 l+1} \\
-q^{3 l+3 / 2}
\end{array} ; q, q\right)=\frac{\left(q^{1 / 2},-q^{3 l+2} ; q\right)_{\infty}}{\left(q,-q^{3 l+3 / 2} ; q\right)_{\infty}}
$$

by (2.5). The right-hand side of (6.14) then simplifies to

$$
\begin{aligned}
& \frac{\left(q^{1 / 2},-q^{2} ; q\right)_{\infty}}{\left(q,-q^{3 / 2} ; q\right)_{\infty}} \sum_{l=0}^{\infty} \frac{(-q ; q)_{3 l}\left(q^{3 / 2} ; q^{3}\right)_{l}}{\left(-q^{2} ; q\right)_{3 l}\left(q^{3} ; q^{3}\right)_{l}} q^{3 l / 2}=\frac{\left(q^{1 / 2},-q^{2} ; q\right)_{\infty}}{\left(q,-q^{3 / 2} ; q\right)_{\infty}} \\
& \quad 2 \phi_{1}\left(\begin{array}{c}
-q, q^{3 / 2} \\
-q^{4}
\end{array} q^{3}, q^{3 / 2}\right) \\
& =\left(1+q^{1 / 2}\right) \frac{\left(q^{1 / 2},-q ; q\right)_{\infty}\left(-q^{5 / 2}, q^{3} ; q^{3}\right)_{\infty}}{\left(q,-q^{1 / 2} ; q\right)_{\infty}\left(-q, q^{3 / 2} ; q^{3}\right)_{\infty}}
\end{aligned}
$$

again by (2.5). The left-hand side of (6.14) is

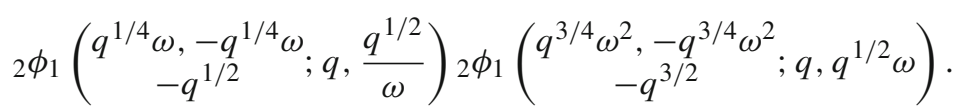

The second factor is computed by (6.1a), with $\omega$ replaced by $\omega^{2}$. After simplification, this results in (6.1b).

Proof of (1.3h). If we let $a=q^{3 / 4}$ in (6.12c), the right-hand side becomes

$$
\frac{\left(-q^{1 / 2} ; q\right)_{\infty}\left(q^{3 / 2} ; q^{6}\right)_{\infty}}{(q ; q)_{\infty}}{ }_{2} \phi_{1}\left(\begin{array}{c}
q^{1 / 2}, q^{9 / 2} \\
q^{13 / 2} ; q^{6}, q^{3 / 2}
\end{array}\right)
$$

Applying the $q$-Gauss summation (2.5) and simplifying gives (6.1c). 
Proof of (1.3i). To prove the equivalent identity (6.1d) we would like to let $a=q^{5 / 4}$ in $(6.12 \mathrm{~d})$. Since the series on the right diverge at this point, we transform them using [8, (III.9)]. This gives

$$
\begin{aligned}
& { }_{2} \phi_{2}\left(\begin{array}{c}
a^{2} q^{-3} \omega, a^{2} q^{-3} \omega^{2} \\
a^{3} q^{-3},-a^{3} q^{-3} ; q,-a^{2} q^{-1}
\end{array}\right)=\frac{\left(1-a^{2} q^{-2}\right)\left(a^{8} q^{-8} ; q^{6}\right)_{\infty}}{\left(1-a^{6} q^{-6}\right)\left(a^{2} q^{-3} ; q\right)_{\infty}\left(a^{4} q^{-3} ; q^{2}\right)_{\infty}} \\
& \times\left(\frac{\left(a^{4} q^{-3} ; q^{6}\right)_{\infty}}{\left(a^{6} q^{-2} ; q^{6}\right)_{\infty}} 3 \phi_{2}\left(\begin{array}{c}
a^{2} q, a^{4} q^{-7}, a^{4} q^{-3} \\
a^{6} q^{-4}, a^{8} q^{-8} ; q^{6}, a^{4} q^{-3}
\end{array}\right)\right. \\
& \left.-a^{2} q^{-3}\left(1-a^{2} q^{-1}\right) \frac{\left(a^{4} q ; q^{6}\right)_{\infty}}{\left(a^{6} q^{-4} ; q^{6}\right)_{\infty}} 3 \phi_{2}\left(\begin{array}{c}
a^{2} q, a^{4} q^{-7}, a^{4} q^{-5} \\
a^{6} q^{-2}, a^{8} q^{-8}
\end{array} ; q^{6}, a^{4} q\right)\right) \text {. }
\end{aligned}
$$

By analytic continuation, this holds for $\left|a^{4} q^{-3}\right|<1$. When $a=q^{5 / 4}$, the ${ }_{3} \phi_{2}$-series on the right reduce to

$$
{ }_{1} \phi_{0}\left(q^{-2} ; q^{6}, q^{2}\right)=0
$$

and

$$
{ }_{3} \phi_{2}\left(\begin{array}{c}
q^{7 / 2}, q^{-2}, 1 \\
q^{11 / 2}, q^{2} ; q^{6}, q^{6}
\end{array}\right)=1
$$

respectively. After simplification, we arrive at (6.1d).

Proof of (5.4). Replace $q$ by $-q^{1 / 2}$ in (6.12b) and then let $a=q^{1 / 4}$. This gives

$$
\begin{aligned}
{ }_{2} \phi_{1}\left(\begin{array}{c}
q^{1 / 4} \omega,-q^{3 / 4} \omega \\
-q
\end{array} q, q^{1 / 2} \omega^{2}\right)= & \frac{\left(q^{3 / 4} ;-q^{1 / 2}\right)_{\infty}\left(-q^{3 / 4} ;-q^{3 / 2}\right)_{\infty}}{\left(q^{1 / 2} \omega^{2}, q ; q\right)_{\infty}} \\
& 2 \phi_{1}\left(\begin{array}{c}
-q^{1 / 4}, q^{3 / 4} \\
q^{7 / 4} ;-q^{3 / 2},-q^{3 / 4}
\end{array}\right) .
\end{aligned}
$$

Applying (2.5) and simplifying we obtain (6.2).

\subsection{Quadratic transformations}

We conclude by discussing some applications of Lemma 6.3 to one-variable series. We will need the generating function $[10,19]$

$$
\frac{(b t z ; q)_{\infty}}{(t z ; q)_{\infty}} 3 \phi_{2}\left(\begin{array}{l}
a, b, a z^{2} \\
a^{2}, b t z
\end{array} ; q, \frac{t}{z}\right)=\sum_{n=0}^{\infty} \frac{(b ; q)_{n}}{\left(a^{2} ; q\right)_{n}} C_{n}(x ; a \mid q) t^{n}, \quad x=\frac{z+z^{-1}}{2}
$$

Comparing it with (6.7) gives the quadratic transformation formulas

$$
{ }_{2} \phi_{1}\left(\begin{array}{c}
a z,-a z \\
-a^{2}
\end{array} ;, \frac{t}{z}\right)=\frac{\left(a^{2} t z q ; q^{2}\right)_{\infty}}{\left(t q / z ; q^{2}\right)_{\infty}} \phi_{2}\left(\begin{array}{c}
a^{2}, a^{2} q, a^{2} z^{2} \\
a^{4}, a^{2} t z q
\end{array} ; q^{2}, \frac{t}{z}\right)
$$




$$
{ }_{2} \phi_{1}\left(\begin{array}{c}
a z^{2}, a z^{2} q \\
a^{2} q
\end{array} q^{2}, \frac{t^{2}}{z^{2}}\right)=\frac{(-a t z ; q)_{\infty}}{(-t / z ; q)_{\infty}} \phi_{2}\left(\begin{array}{c}
a,-a, a z^{2} \\
a^{2},-a t z
\end{array} ; q, \frac{t}{z}\right) .
$$

The identity (6.15b) is due to Jain [12]. Although we would be surprised if (6.15a) were new, we could not find it in the literature.

A pair of closely related identities were obtained by Gessel and Stanton [9, Eqs. (5.6), (5.18)], namely,

$$
\begin{aligned}
{ }_{2} \phi_{1}\left(\begin{array}{c}
a,-a \\
-c
\end{array} ; q, c x\right) & \doteq \frac{\left(a^{2} x ; q^{2}\right)_{\infty}}{\left(x ; q^{2}\right)_{\infty}} 3 \phi_{2}\left(\begin{array}{c}
c, c q, a^{2} \\
c^{2}, q^{2} / x
\end{array} ; q^{2}, q^{2}\right), \\
{ }_{2} \phi_{1}\left(\begin{array}{c}
a, a q \\
c^{2} q
\end{array} q^{2}, c^{2} x^{2}\right) & \doteq \frac{(a x ; q)_{\infty}}{(x ; q)_{\infty}} 3 \phi_{2}\left(\begin{array}{l}
c,-c, a \\
c^{2}, q / x
\end{array} ;, q\right) .
\end{aligned}
$$

Here, $\doteq$ means identity as formal power series in $x$. Although the right-hand sides converge for generic values of the parameters, they have poles accumulating at $x=0$, so the corresponding power series diverge. Thus, (6.16) do not hold as identities between convergent series (and the authors of [9] make no such claim). However, applying [8, III.34] to (6.15) and changing parameters, one obtains

$$
\begin{aligned}
& { }_{2} \phi_{1}\left(\begin{array}{l}
a,-a \\
-c
\end{array} ; q, c x\right)=\frac{\left(a^{2} x ; q^{2}\right)_{\infty}}{\left(x ; q^{2}\right)_{\infty}} 3 \phi_{2}\left(\begin{array}{l}
c, c q, a^{2} \\
c^{2}, q^{2} / x
\end{array} ; q^{2}, q^{2}\right) \\
& +\frac{\left(a^{2}, c^{2} x ; q^{2}\right)_{\infty}}{(-c, c x ; q)_{\infty}\left(1 / x ; q^{2}\right)_{\infty}} 3 \phi_{2}\left(\begin{array}{c}
c x, c q x, a^{2} x \\
c^{2} x, q^{2} x
\end{array} ; q^{2}, q^{2}\right), \\
& { }_{2} \phi_{1}\left(\begin{array}{c}
a, a q \\
c^{2} q
\end{array} q^{2}, c^{2} x^{2}\right)=\frac{(a x ; q)_{\infty}}{(x ; q)_{\infty}}{ }_{3} \phi_{2}\left(\begin{array}{c}
c,-c, a \\
c^{2}, q / x
\end{array} ; q, q\right) \\
& +\frac{\left(a, c^{2} x ; q\right)_{\infty}}{\left(c^{2} q, c^{2} x^{2} ; q^{2}\right)_{\infty}(1 / x ; q)_{\infty}} 3 \phi_{2}\left(\begin{array}{c}
c x,-c x, a x \\
c^{2} x, q x
\end{array} ; q, q\right) \text {. }
\end{aligned}
$$

These identities can be viewed as analytic versions of the formal identities (6.16).

Finally, we note that an interesting identity is obtained by substituting $z=\mathrm{i}$ in (6.7a) and using [8, Ex. (7.17)]

$$
C_{n}(0 ; a \mid q)= \begin{cases}(-1)^{k} \frac{\left(a^{2} ; q^{2}\right)_{k}}{\left(q^{2} ; q^{2}\right)_{k}}, & n=2 k \\ 0, & n=2 k+1\end{cases}
$$

After replacing $a$ by $\mathrm{i} a$ and $t$ by it, we obtain

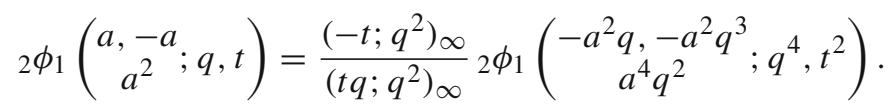

This quartic transformation can also be proved by combining the two identities (6.15). It can be obtained from [4, Eq. (3.19)] by replacing $a$ by $a q^{-n}$ and letting $n \rightarrow \infty$. 
Acknowledgements I would like to thank Chris Jennings-Shaffer, Jeremy Lovejoy and Ole Warnaar for useful comments on the manuscript.

Funding Open Access funding provided by Chalmers University of Technology

Open Access This article is licensed under a Creative Commons Attribution 4.0 International License, which permits use, sharing, adaptation, distribution and reproduction in any medium or format, as long as you give appropriate credit to the original author(s) and the source, provide a link to the Creative Commons licence, and indicate if changes were made. The images or other third party material in this article are included in the article's Creative Commons licence, unless indicated otherwise in a credit line to the material. If material is not included in the article's Creative Commons licence and your intended use is not permitted by statutory regulation or exceeds the permitted use, you will need to obtain permission directly from the copyright holder. To view a copy of this licence, visit http://creativecommons.org/licenses/by/4.0/.

\section{References}

1. Andrews, G.E.: Schur's theorem, Capparelli's conjecture and $q$-trinomial coefficients. In: Andrews, G.E., Bressoud, D.M., Parson, L.A. (eds.) The Rademacher Legacy to Mathematics, pp. 141-154. American Mathematical Society, Providence (1994)

2. Askey, R., Ismail, M.E.H.: A generalization of ultraspherical polynomials. In: Erdős, P. (ed.) Studies in Pure Mathematics, pp. 55-78. Birkhäuser, Basel (1983)

3. Askey, R., Wilson, J.: Some basic hypergeometric orthogonal polynomials that generalize Jacobi polynomials. Mem. Am. Math. Soc. 54, 1-8 (1985)

4. Berkovich, A., Warnaar, S.O.: Positivity preserving transformations for $q$-binomial coefficients. Trans. Am. Math. Soc. 357, 2291-2351 (2005)

5. Bringmann, K., Jennings-Shaffer, C., Mahlburg, K.: Proofs and reductions of various conjectured partition identities of Kanade and Russell. J. Reine Angew. Math. 766, 109-135 (2020)

6. Capparelli, S.: On some representations of twisted affine Lie algebras and combinatorial identities. J. Algebra 154, 335-355 (1993)

7. Dousse, J., Lovejoy, J.: Generalizations of Capparelli’s identity. Bull. Lond. Math. Soc. 51, 193-206 (2019)

8. Gasper, G., Rahman, M.: Basic Hypergeometric Series, $2^{\text {nd }}$ Edition. Cambridge University Press, Cambridge (2004)

9. Gessel, I., Stanton, D.: Applications of $q$-Lagrange inversion to basic hypergeometric series. Trans. Am. Math. Soc. 277, 173-201 (1983)

10. Ismail, M.E.H., Masson, D.R., Suslov, S.K.: Some generating functions for $q$-polynomials. In: Ismail, M.E.H., Masson, D.R., Rahman, M. (eds.) Special Functions, $q$-Series and Related Topics, pp. 91-108. American Mathematical Society, Providence (1997)

11. Ismail, M.E.H., Wilson, J.A.: Asymptotic and generating relations for the $q$-Jacobi and ${ }_{4} \phi_{3}$ polynomials. J. Approx. Theory 36, 43-54 (1982)

12. Jain, V.K.: Some transformations of basic hypergeometric functions. II. SIAM J. Math. Anal. 12, 957-961 (1981)

13. Kanade, S., Russell, M.C.: Staircases to analytic sum-sides for many new integer partition identities of Rogers-Ramanujan type. Electron. J. Combin. 26, 1-6 (2019)

14. Kurşungöz, K.: Andrews-Gordon type series for Capparelli's and Göllnitz-Gordon identities. J. Combin. Theory A 165, 117-138 (2019)

15. Lepowsky, J., Milne, S.: Lie algebraic approaches to classical partition identities. Adv. Math. 29, 15-59 (1978)

16. Lepowsky, J., Wilson, R.L.: A Lie theoretic interpretation and proof of the Rogers-Ramanujan identities. Adv. Math. 45, 21-72 (1982)

17. Lepowsky, J., Wilson, R.L.: The structure of standard modules. I. Universal algebras and the RogersRamanujan identities. Invent. Math. 77, 199-290 (1984)

18. Misra, K.C.: Specialized characters for affine Lie algebras and the Rogers-Ramanujan identities. In: Andrews, G.E., et al. (eds.) Ramanujan Revisited, pp. 85-109. Academic Press, Boston (1988) 
19. Rahman, M., Verma, A.: A $q$-integral representation of Rogers' $q$-ultraspherical polynomials and some applications. Constr. Approx. 2, 1-10 (1986)

20. Ramanujan, S., Rogers, L.J.: Proof of certain identities in combinatory analysis. Proc. Cambridge Philos. Soc. 19, 214-216 (1919)

21. Rogers, L.J.: Second memoir on the expansion of certain infinite products. Proc. Lond. Math. Soc. 25, 318-343 (1894)

22. Rogers, L.J.: Third memoir on the expansion of certain infinite products. Proc. Lond. Math. Soc. 26, 15-32 (1895)

23. Rosengren, H.: New proofs of determinant evaluations related to plane partitions. Electronic J. Combin. 19, 15 (2012)

24. Watson, G.N.: Theorems stated by Ramanujan (VII): theorems on continued fractions. J. Lond. Math. Soc. 4, 39-48 (1929)

Publisher's Note Springer Nature remains neutral with regard to jurisdictional claims in published maps and institutional affiliations. 